\title{
Nephrectomy Avoided in a Patient with Lipid Poor Angiomylipoma with Radiological Features Suspicious for Renal Cell Carcinoma: A Case Report*
}

\author{
Peter Grice, Rosemina Ahmad, Masood Khan \\ Leicester General Hospital, University Hospitals of Leicester NHS Trust, Leicester, UK \\ Email: peter.grice@nhs.net
}

How to cite this paper: Grice, P., Ahmad, R. and Khan, M. (2018) Nephrectomy Avoided in a Patient with Lipid Poor Angiomylipoma with Radiological Features Suspicious for Renal Cell Carcinoma: A Case Report. Open Journal of Urology, 8, 59-66.

https://doi.org/10.4236/oju.2018.83007

Received: February 20, 2018

Accepted: March 24, 2018

Published: March 27, 2018

Copyright (c) 2018 by authors and Scientific Research Publishing Inc. This work is licensed under the Creative Commons Attribution International License (CC BY 4.0).

http://creativecommons.org/licenses/by/4.0/

\begin{abstract}
Angiomyolipoma (AML) is a benign neoplasm that is easily mistaken for renal cell carcinoma (RCC) upon radiological investigation. The management of the two pathologies is significantly different, and so accurate diagnosis is vital. We report a case in which ultrasound guided biopsy saved the patient from an unnecessary nephrectomy when an AML had radiological appearances consistent with RCC. This case report also discusses the literature surrounding radiological features and novel imaging techniques of minimal-fat AML and RCC.
\end{abstract}

\section{Keywords}

Angiomyolipoma, Minimal-Fat AML, Renal Cell Carcinoma, MRI

\section{Introduction}

Angiomyolipoma (AML) is the most common solid, benign renal neoplasm that clinicians encounter [1]. AML is described as "triphasic" as it is formed from three constituents to varying degrees: adipose tissue, smooth muscle and dysmorphic blood vessels [1]. The presence of large amounts of adipose tissue assists in the radiological diagnosis of "classic" AML [1] [2]. Where there is minimal fat, or fat is masked by intramural haemorrhage, there can be radiological diagnostic difficulty, and AML can be mistaken for renal cell carcinoma (RCC) [1] [3].

AMLs are now understood to be a heterogenous group of tumours with vary${ }^{\star}$ The patient in this case report gave consent for her case to be published. 
ing natural history, pathology and imaging characteristics [1]. Here, we present a case where a potentially unnecessary nephrectomy was avoided as histology from ultrasound-guided biopsy showed AML, while cross-sectional imaging demonstrated a small solid peripelvic renal tumour suspicious for RCC. Confusion arose due to the lipid-poor nature of the lesion, the central position and irregular tumour margins.

\section{Presentation}

A 77-year-old lady presented with generalised, colicky abdominal pain and was admitted under the general surgeons. She had not noticed any visible haematuria. She had a past medical history of type 2 diabetes, hypertension, hypothyroidism and hypercholesterolaemia. On examination of the abdomen, she had generalised tenderness but no guarding or peritonism. She had a normal urine dipstick result and blood haematology and biochemistry revealed normal renal and liver function as well as normal inflammatory markers.

She underwent a contrast-enhanced CT of the abdomen and pelvis which demonstrated adhesions, no evidence of bowel obstruction, and an incidental mass in the left renal pelvis (see Figure 1 and Figure 2). This was reported as a

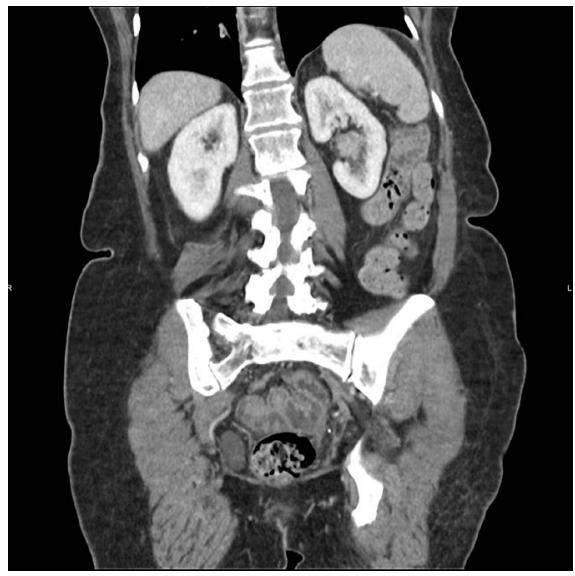

Figure 1. Coronal section of contrast CT demonstrating left renal lipid-poor AML.

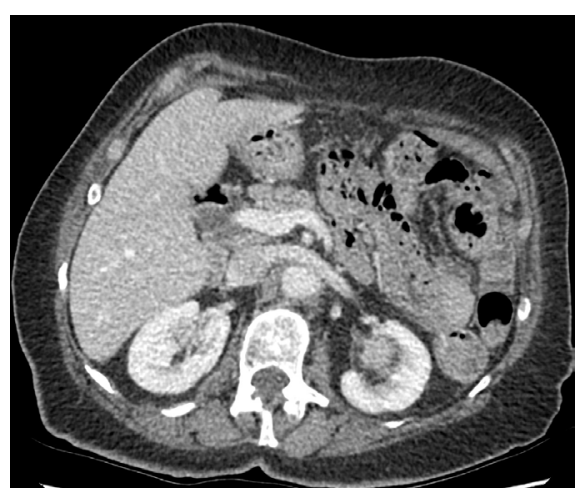

Figure 2. Axial section of contrast CT demonstrating left renal lipid-poor AML. 
$1.9 \mathrm{~cm}$ ill-defined soft tissue mass. The CT also demonstrated multiple sub-centimetre lesions in the spleen and liver and the possibility of metastasis was reported. Due to the location of the renal lesion, the initial radiological concern was that this CT was likely to be consistent with an upper tract transitional cell carcinoma (TCC). She was referred to urology multidisciplinary team meeting (MDT) and discharged from the surgical team.

A week later, an outpatient MRI demonstrated that the liver and splenic lesion were simple cysts rather than metastatic disease, although the renal lesion was restricting diffusion and enhancing on post contrast arterial phase with venous phase washout, which were reported as features consistent with an upper tract TCC (see Figures 3-7). She also underwent a CT of her chest, which did not show any abnormality. She was seen in clinic a week later with those results and a plan was made for elective left flexible ureteroscopy, retrograde studies and ureteric JJ stent insertion. This was performed six weeks post original presentation and did not detect renal pelvis or collecting system lesion and no other abnormality was identified.

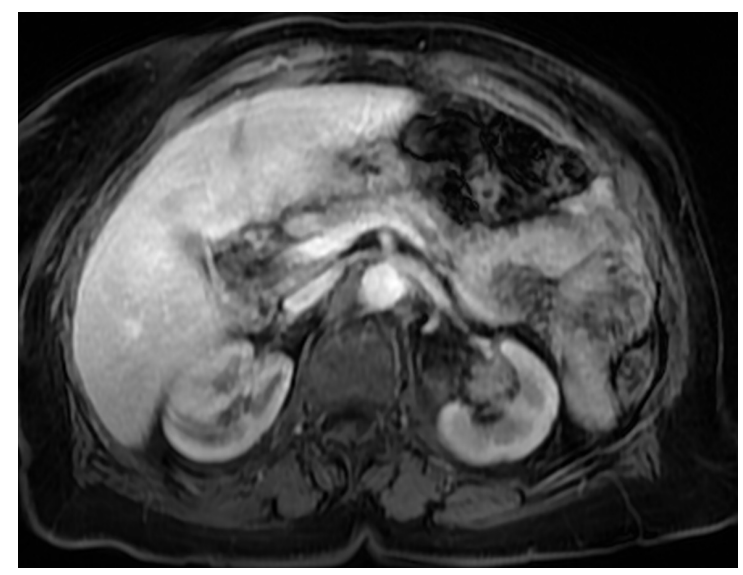

Figure 3. Axial section of contrast enhanced sequence demonstrating enhancement in the lipid-poor AML.

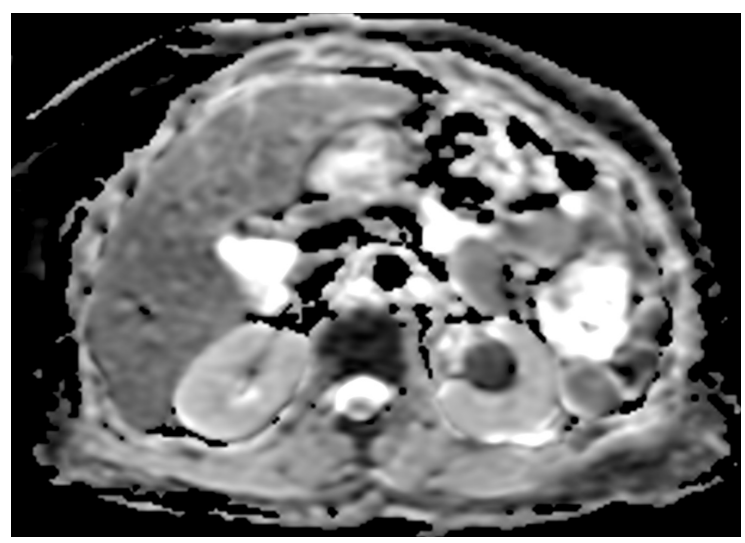

Figure 4. Apparent diffusion coefficient mean apparent propagator MRI demonstrating restricted diffusion in the lipid poor AML. 


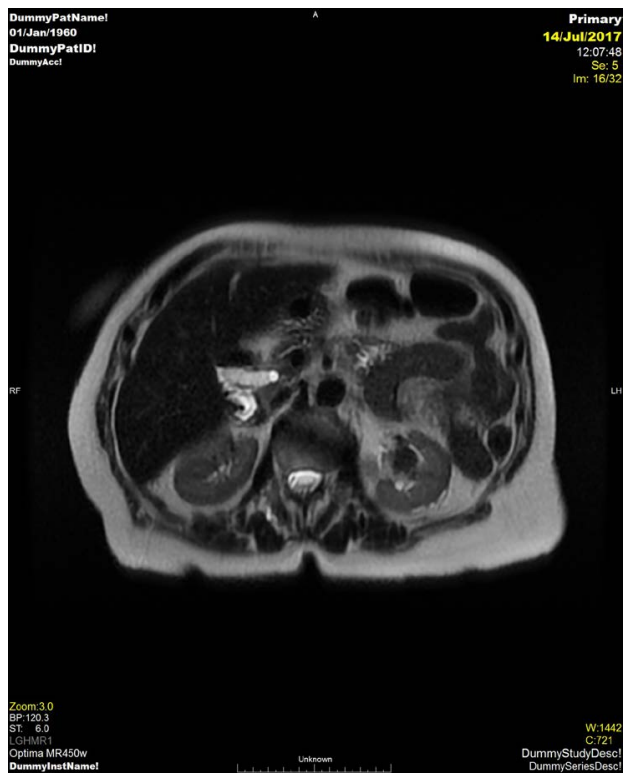

Figure 5. Low T2 signal on MRI compared with renal cortex makes clear cell RCC less likely.

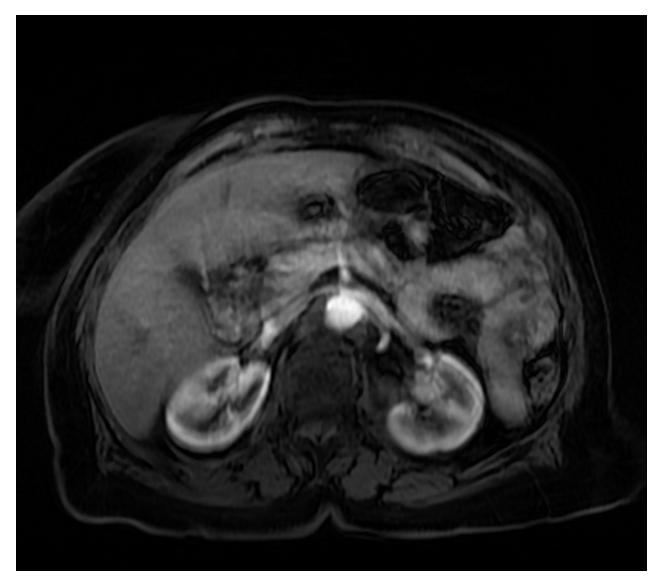

Figure 6. Cortico-medullary series MR demonstrating prominent and prolonged enhancement in cortico-medullary phase. This is not typical for papillary RCC.

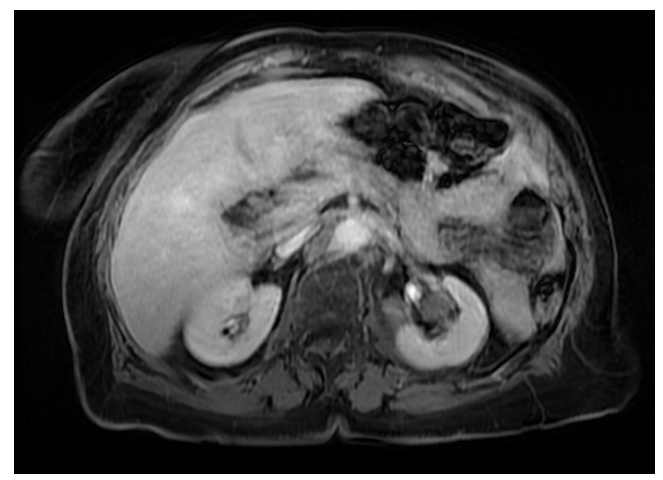

Figure 7. Nephrographic phase ten minutes later. 
She was reviewed in clinic two weeks later with an interim urology MDT discussion. During MDT, images were reviewed, and the working diagnosis changed from upper tract TCC to RCC. She continued to be asymptomatic and denied any visible haematuria. As the diagnosis was not clear at this stage, the MDT decision was that she should undergo ultrasound-guided biopsy of the left renal lesion, rather than proceeding to nephroureterectomy. This was performed two weeks later and the patient was reviewed shortly afterwards with the histology result. Surprisingly, the histology demonstrated AML, not RCC in Figure 8. A week later, her stent was removed without complication. There was no growth in the AML following six month interval surveillance and she has now been discharged. She has been advised that unless she becomes symptomatic, no further follow up or intervention is required.

\section{Discussion}

There are other cases in the literature in which AML and RCC are mistaken for each other. AMLs occur sporadically in $80 \%$ of cases, while $20 \%$ of AMLs are associated with Tuberous Sclerosis (TS). Obuz et al. described a case of TS in which a 21-year-old woman presented with flank pain and abdominal distension. CT and MRI demonstrated multiple bilateral renal lesions, all but one of which had radiological features consistent with AML. The other lesion had no detectable fat content and was very characteristic of RCC. The patient went on to have nephrectomy, with the histology demonstrating that all lesions were, in fact, AML [4]. A similar case was reported by Itano et al. Once again, a renal lesion with very little adipose content in a TS patient was found to be AML rather than RCC, which was the suspicion prior to nephrectomy [5].

Nakamura et al., described a case in which a 40-year-old man with TS presented with left flank mass. Contrast CT demonstrated irregularly enhanced masses in upper pole and interpolar regions of the left kidney and bilateral renal nodules. Left renal angiography demonstrated hypervascular areas in the left

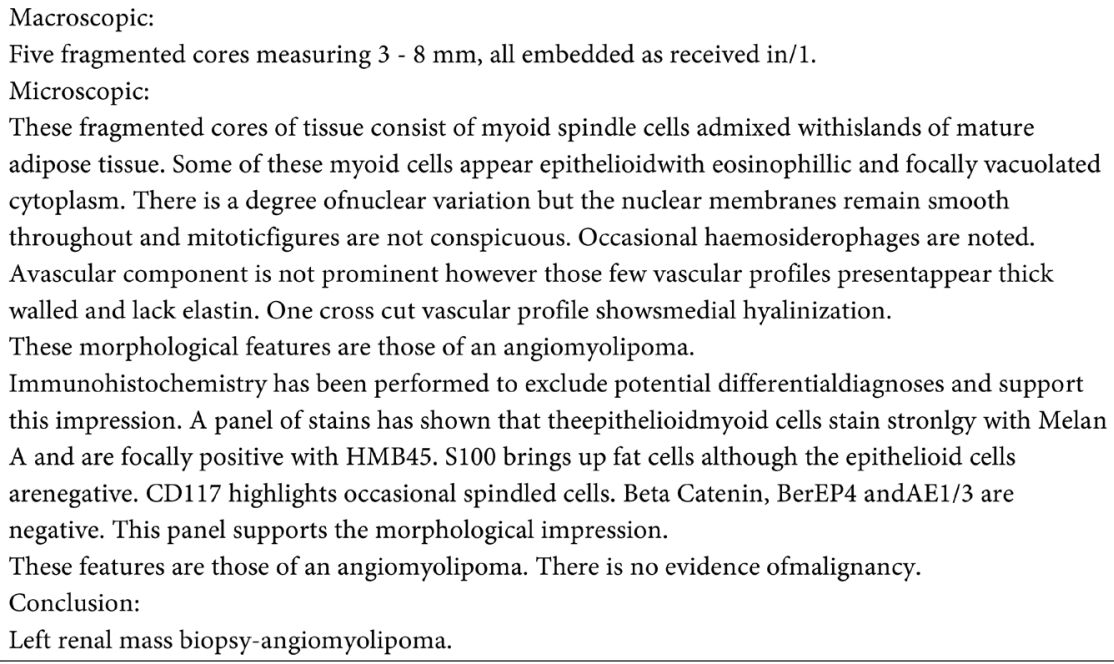

Figure 8. Histology report demonstrating AML. 
upper pole and interpolar region. These findings were deemed to be consistent with RCCs with concurrent AML and these findings were confirmed on histopathological analysis [6].

In 1989, Yasukawa et al. described a case in which what was thought to be large AML was found to be RCC. An 18-year-old woman with TS was investigated for a large left renal mass and bilateral smaller renal masses with varying degrees of adipose content. Angiography confirmed bilateral hypervascular lesions. The working diagnosis was bilateral AMLs. The patient underwent left nephrectomy, removing a $4750 \mathrm{~g}$ specimen. This was found to be RCC on histological examination [7].

Lipid rich AMLs typically demonstrate a measurable lipid component, and are often associated with a renal cortical defect on cross sectional imaging. Diagnostic difficulty can arise in lipid poor AMLs, particularly if less than $3 \mathrm{~cm}$, due to difficulty in accurate detection of intra-lesional fat and variable appearance. Clear cell RCCs can have increased levels of intracellular fat and mimic lipid poor AMLs.

There have been many papers exploring imaging features of minimal fat AML. Sung et al., compared morphology and enhancement features in sized matched AML and RCC. It was concluded that, in CT imaging, "non-round shape" in the absence of capsule and prolonged enhancement were factors that could distinguish minimal-fat AML from RCC [8]. Prolonged enhancement was also found to be an important radiological feature in distinguishing the two pathologies by Kim et al. [9].

Zhang et al. performed a retrospective study comparing the imaging and post-operative histology of minimal fat AML to RCC. Each patient had a pre-operative helical CT, which were reported by two blinded radiologists. The study found that unenhanced attenuation characteristics, intra-tumoural vessels and the attenuation values of unenhanced and early excretory phase scans were useful features to distinguish between the two pathologies [10].

The MRI signal intensity and enhancement characteristics can also be helpful in the diagnosis of lipid-poor AMLs. Low signal intensity on T2 weighted imaging compared to the signal intensity of the renal cortex favours a diagnosis of lipid-poor AML rather than clear cell RCC [11]. However, papillary RCCs can also have low T2 signal intensity but these lesions typically have a lower level enhancement which is less prolonged than AMLs [12].

Several factors confounded the diagnostic interpretation of the lipid-poor renal AML in this case; the central peripelvic position which raised the possibility of an upper tract TCC, irregular margins on CT and absence of discernible intra-tumoural fat. However, on review of the MRI characteristics for a renal mass the T2 high signal intensity and prolonged enhancement favoured a lipid-poor AML rather than RCC. With appropriate facilities and expertise in place, the MDT took the decision to obtain histology. In retrospect, this was a wise decision, as the histology showed classical features of AML and, therefore, a neph- 
rectomy was avoided.

\section{Conclusion}

Despite advances in radiological practice particularly with development of multiparametric MRI techniques, there can still be uncertainty regarding the nature of renal tumours, particularly if less than $3 \mathrm{~cm}$. In these cases, histology should be obtained from either ureteroscopy or percutaneous biopsy if the diagnosis remains uncertain. This can avoid unnecessary nephrectomy and the consequential associated morbidity and mortality.

\section{References}

[1] Jinzaki, M., Silverman, S.G., Akita, H., Nagashima, Y., Mikami, S. and Oya, M. (2014) Renal Angiomyolipoma: A Radiological Classification and Update on Recent Developments in Diagnosis and Management. Abdominal Imaging, 39, 588-604. https://doi.org/10.1007/s00261-014-0083-3

[2] Lane, B.R., Aydin, H., Danforth, T.L., Zhou, M., Remer, E.M., Novick, A.C. and Campbell, S.C. (2008) Clinical Correlates of Renal Angiomyolipoma Subtypes in 209 Patients: Classic, Fat Poor, Tuberous Sclerosis Associated and Epithelioid. Journal of Urology, 180, 836-843. https://doi.org/10.1016/j.juro.2008.05.041

[3] Lienert, A.R. and Nicol, D. (2012) Renal Angiomyolipoma. BJU International, 110, 25-27. https://doi.org/10.1111/j.1464-410X.2012.11618.x

[4] Obuz, F., Karabay, N., Seçil, M., Igci, E., Kovanlikaya, A. and Yörükoglu, K. (2000) Various Radiological Appearances of Angiomyolipomas in the Same Kidney. European Radiology, 10, 897-899. https://doi.org/10.1007/s003300051031

[5] Itano, S., Sakai, T., Ijuin, H., Koga, K., Yoshiyama, Y., Yoshida, T., Kajiwara, M., Ono, N., Noguchi, H., Hirai, K. and Tanikawa, K. (1997) Three Cases of Renal Angiomyolipoma. Internal Medicine, 36, 206-210. https://doi.org/10.2169/internalmedicine.36.206

[6] Nakamura, Y., Murayama, K., Katsumi, T., Watanabe, K., Kishitani, M. and Ohkawa, M. (1994) Coincident Renal Cell Carcinoma and Renal Angiomyolipoma in Tuberous Sclerosis: A Case Report. Hinyokika Kiyo, 40, 703-706.

[7] Yasukawa, S., Aoshi, H., Kyoku, I., Uekado, Y. and Ohkawa, T. (1989) Renal Cell Carcinoma Associated with Tuberous Sclerosis: A Case Report. Hinyokika Kiyo, 35, 2135-2138.

[8] Sung, C.K., Kim, S.H., Woo, S., Moon, M.H., Kim, S.Y., Kim, S.H. and Cho, J.Y. (2016) Angiomyolipoma with Minimal Fat: Differentiation of Morphological and Enhancement Features from Renal Cell Carcinoma at CT Imaging. Acta Radiologica, 57, 1114-1122. https://doi.org/10.1177/0284185115618547

[9] Kim, J.K., Park, S.Y. and Shon, J.H. (2004) Angiomyolipoma with Minimal Fat: Differentiation from Renal Cell Carcinoma at Biphasic Helical CT. Radiology, 230, 677-684. https://doi.org/10.1148/radiol.2303030003

[10] Zhang, Y.Y., Luo, S., Liu, Y. and Xu, R.T. (2013) Angiomyolipoma with Minimal Fat: Differentiation from Papillary Renal Cell Carcinoma by Helical CT. Clinical Radiology, 68, 365-570. https://doi.org/10.1016/j.crad.2012.08.028

[11] Hindman, N., et al. (2012) Angiomyolipoma with Minimal Fat: Can It Be Differentiated from Clear Cell Carcinoma by Using Standard MR Techniques? Radiology, 265, 468-477. https://doi.org/10.1148/radiol.12112087 
[12] Canvasser, N., et al. (2017) Diagnostic Accuracy of Multiparametric Magnetic Resonance Imaging to Identify Clear Cell Renal Carcinoma in cT1a Renal Masses. The Journal of Urology, 198, 780-786. https://doi.org/10.1016/j.juro.2017.04.089 